\title{
Evaluation of Marginal Adaptation of Composite Restorations Reinforced with Novel Enamel Inserts (Biofillers) in Class V Cavities
}

\author{
Nandini Biradar ${ }^{1}$, Keerti S Allappanavar ${ }^{2}$, Nithin K Shetty ${ }^{3}$, Basanagouda S Patil ${ }^{4}$, Reshma S Hegde ${ }^{5}$, Prashant Moogi ${ }^{6}$
}

\begin{abstract}
Aim and objective: To evaluate the marginal adaptation at the tooth-restoration interface at enamel and cementum margins using composite restoration reinforced with novel enamel inserts/biofillers.

Materials and methods: Standardized class V box-shaped cavities were prepared in 40 extracted maxillary first premolar teeth which were divided randomly into four experimental groups consisting of 10 samples each. Group I: Bulk placement. Groups II: Horizontal incremental technique. Group III: Restoration with precured composite balls (megafillers). Group IV: Restoration with biofillers. All the cavities were restored with visible light-activated direct restorative nanocomposite. The specimens were thermocycled for 24 hours. After thermocycling, the samples were immersed in a $1 \%$ methylene blue for 4 hours and subsequently evaluated for microleakage. Microleakage scores (0-4) were obtained from gingival margins of class $V$ restorations and analyzed by statistical analysis. Evaluation of the data was performed by Kruskal-Wallis one-way analysis of variance (ANOVA), and Mann-Whitney $U$ tests.

Results: Microleakage scores have indicated restorations with biofillers showed best results followed by megafillers, incremental horizontal build-up, and bulk filling.

Conclusion: Biofillers provide a novel approach in improving microleakage and marginal adaptability of composite resin restorations.

Clinical significance: Incorporation of inserts, which are capable of adequate bonding to resin and tooth, may provide improved marginal adaptability and reduce microleakage around restorative margins.

Keywords: Biofillers, Enamel inserts, Megafillers polymerization shrinkage, Microleakage.

The Journal of Contemporary Dental Practice (2020): 10.5005/jp-journals-10024-2964
\end{abstract}

\section{INTRODUCTION}

The ideal class $V$ restorative for clinical use should have adequate retention/bonding to tooth structure, resistance to fracture, easy to use, and have good longevity. Most commonly used restorative materials indicated in class $\mathrm{V}$ restorations are glass ionomer cements, resin-modified glass ionomer cements, and composite resins. Composites are the first choice of restoration due to their superior esthetic and mechanical properties. Longevity of resin composites is often difficult in cervical lesions owing to complex tooth microstructure, local stress concentration, and wet restorative environment. Polymerization shrinkage remains the major drawback of composite resin restorations. Composite restorations often fail due to microleakage and poor marginal adaptation as a result of their inherent deficiency of polymerization shrinkage. ${ }^{1}$ Marginal adaptation is defined as the interfacial distance between the eluted restoration and the tooth structure. The marginal adaptation of composite resins can be improved by decreasing polymerization shrinkage. Of the various methods used to reduce polymerization shrinkage of composite resins, filler loading is widely accepted and several fillers have been incorporated into composite resins. Increased filler-resin ratio counters polymerization shrinkage but adversely affects working properties of a composite. ${ }^{2}$ Alternatively, megafillers/inserts have been implicated in reducing composite volume by $50-75 \%$, with a concomitant reduction in polymerization shrinkage and marginal microleakage. ${ }^{3}$ The
${ }^{1}$ Department of Dentistry, Bidar Institute of Medical Sciences, Bidar, Karnataka, India

${ }^{2}$ Department of Conservative Dentistry and Endodontics, SB Patil Institute for Dental Sciences and Research, Bidar, Karnataka, India

${ }^{3}$ Department of Conservative Dentistry and Endodontics, MS Ramaiah University of Applied Sciences, Bengaluru, Karnataka, India

${ }^{4}$ Department of Conservative Dentistry and Endodontics, PM Nadagouda Memorial Dental College and Hospital, Bagalkot, Karnataka, India

${ }^{5}$ Department of Conservative Dentistry and Endodontics, Speciality Dental Clinic and Orthodontic Centre, Cleveland, Bengaluru, Karnataka, India

${ }^{6}$ Department of Conservative Dentistry and Endodontics, Yogita Dental College and Hospital, Khed, Ratnagiri, Maharashtra, India

Corresponding Author: Keerti S Allappanavar, Department of Conservative Dentistry and Endodontics, SB Patil Institute for Dental Sciences and Research, Bidar, Karnataka, India, Phone: +91 9739052023, e-mail: drkeerti.s.a@gmail.com

How to cite this article: Biradar N, Allappanavar KS, Shetty NK, et al. Evaluation of Marginal Adaptation of Composite Restorations Reinforced with Novel Enamel Inserts (Biofillers) in Class V Cavities. J Contemp Dent Pract 2020;21(12):1368-1373.

Source of support: Nil

Conflict of interest: None

(c) Jaypee Brothers Medical Publishers. 2020 Open Access This article is distributed under the terms of the Creative Commons Attribution 4.0 International License (https://creativecommons.org/licenses/by-nc/4.0/), which permits unrestricted use, distribution, and non-commercial reproduction in any medium, provided you give appropriate credit to the original author(s) and the source, provide a link to the Creative Commons license, and indicate if changes were made. The Creative Commons Public Domain Dedication waiver (http://creativecommons.org/publicdomain/zero/1.0/) applies to the data made available in this article, unless otherwise stated. 
anatomy and flexural stresses in cervical area further challenge the retention and adaptation of composite restorations in class $\mathrm{V}$ cavities, where microleakage tends to be higher. ${ }^{4}$ Cervical lesions pose a significant challenge to retain composite resin restorations demanding appropriate restorative material, technique, and skill. Structurally weaker cervical enamel and dentin which are subjected to higher tensile loads further complicate the longevity of composite resins. Thus, long-term retention of composite resin restoration in class $V$ cavities is challenging, requiring meticulous planning for appropriate restorative material and technique. Hence, this article evaluates the marginal adaptation of class $\mathrm{V}$ nanocomposite restorations restored with reinforced novel enamel inserts/biofillers, compares it with the following three methods; bulk, horizontal incremental, precured composite balls as inserts in reducing microleakage in class $V$ nanocomposite restoration.

\section{Materials and Methods}

The study was carried out in vitro in the Department of Conservative Dentistry and Endodontics, at K.L.E. Dental College and Hospital, Bengaluru, Karnataka. Extracted teeth were obtained from the department of Oral and Maxillofacial Surgery from the same institute. Stereomicroscopic evaluation was performed at Oxford Dental College, Bengaluru, Karnataka.

\section{Specimen Preparation}

Sample size $(N=40)$ of 40 was selected as suggested by SPSS software. Forty caries free, maxillary first premolar teeth without any developmental anomalies, stains, cracks, or fracture, which were planned and extracted for orthodontic treatments, were collected from the Department of Oral and Maxillofacial Surgery. The teeth samples were ultrasonically cleaned by a scaler to remove debris, calculus, and stored in distilled water. Standardized class V boxshaped cavities (Fig. 1) were prepared in all 40 using diamond points with aerator handpiece. (Standard dimensions of class $V$ cavities; $2.5 \mathrm{~mm}$ depth pulpally, $3 \mathrm{~mm}$ height occluso-gingivial, and $3.5 \mathrm{~mm}$ width mesiodistally. Gingival floor was kept above CEJ.) Following cavity preparation, all the 40 teeth were randomly allocated into 4 groups of 10 samples each.

\section{Acid-etching, Bonding, and Restoration of Class V Cavities}

All the 40 standard cavities were etched with $37 \%$ phosphoric acid (3M ESPE) for 15 seconds, washed with distilled water for 10

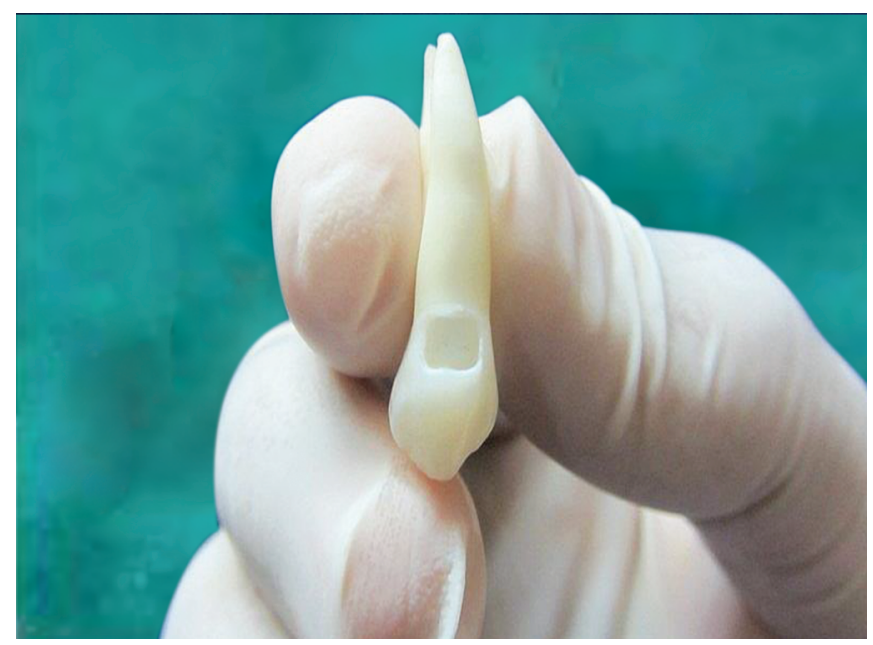

Fig. 1: Standardized class V cavity preparation seconds, and dried with a gentle blast of air. Caution was taken not to overdry the preparations. Two coats of bonding agent (prime and bond, DENTSPLY) were applied at intervals of 10 seconds and cured for 10 seconds. Restorations were carried out according to the following four study groups:

\section{Group I: $(n=10)$ Bulk Filling}

Cavities received a single layer of nanocomposite (Filtek ${ }^{\mathrm{TM}} \mathrm{Z} 3503 \mathrm{M}$ ESPE) till the cavosurface margin and the increment was cured for 40 seconds. Subsequently finished and polished with composite polishing kit (Shofu Inc., Kyoto, Japan).

\section{Group II: $(n=10)$ Horizontal Incremental Technique}

Cavities received placement of nanocomposite (Filtek ${ }^{\mathrm{TM}}$ Z350 3M ESPE) in horizontal increments of approximately $1.5 \mathrm{~mm}$ and each increment was cured for 20 seconds. Two increments were placed. Subsequently finished and polished with composite polishing kit (Shofu Inc., Kyoto, Japan).

\section{Group III: $(n=10)$ Restoration with Precured Composite Balls (Megafillers) \\ Fabrication of Precured Composite Balls}

Precured composite balls were made using a silicone mold consisting of $2.5 \mathrm{~mm}$ width and $2 \mathrm{~mm}$ height, into which the nanocomposite was packed and cured for 40 seconds.

\section{Restoration of Class V Cavities}

Nanocomposite (Filtek ${ }^{\text {TM }}$ Z350 3M ESPE) of $1 \mathrm{~mm}$ of horizontal increment was placed in the cavities and left uncured. Precured composite ball inserts were embedded into the cavities containing uncured nanocomposite. It was ensured that the inserts were adequately covered by nanocomposite on surface. Excess resin was trimmed off with a scalpel. Curing was performed in all the samples from all directions for 40 seconds. Subsequently finished and polished with composite polishing kit (Shofu Inc., Kyoto, Japan).

\section{Group IV: $(n=10)$ Restoration with Enamel Inserts (Biofillers)}

\section{Fabrication of Biofiller (Fig. 2)}

Five, intact maxillary premolar teeth extracted for orthodontic treatment with adequate color, form, and dimensions were selected. Crowns were sectioned at the occlusal third into dimensions of 3 $\times 2 \mathrm{~mm}$ sections to form fillers consisting of only enamel. These fillers were autoclaved at a temperature of $121^{\circ} \mathrm{C}$, for 15 minutes. Fillers were etched with $37 \%$ phosphoric acid for 15 seconds. Rinsed with distilled water for 20 seconds. Dried with a gentle jet of air, coated with an adhesive system prime and bond (DENTSPLY), dried again for 20 seconds with a jet of air and light polymerized for 10 seconds as recommended by the manufacturer. Thus, biofillers were prepared (Enamel Inserts).

\section{Restoration of Class V Cavities}

Nanocomposite (Filtek ${ }^{\mathrm{TM}}$ Z350 3M ESPE) of $1 \mathrm{~mm}$ of horizontal increment was placed in the cavities and left uncured. Dentin bonding agent coated biofillers (Enamel inserts) were embedded into the cavity containing uncured nanocomposite. It was ensured that the enamel inserts were adequately covered by nanocomposite on surface. Excess resin flash was trimmed with a scalpel. Curing was performed in all the samples from all directions for 40 seconds. Subsequently finished and polished with composite polishing kit (Shofu Inc., Kyoto, Japan). 

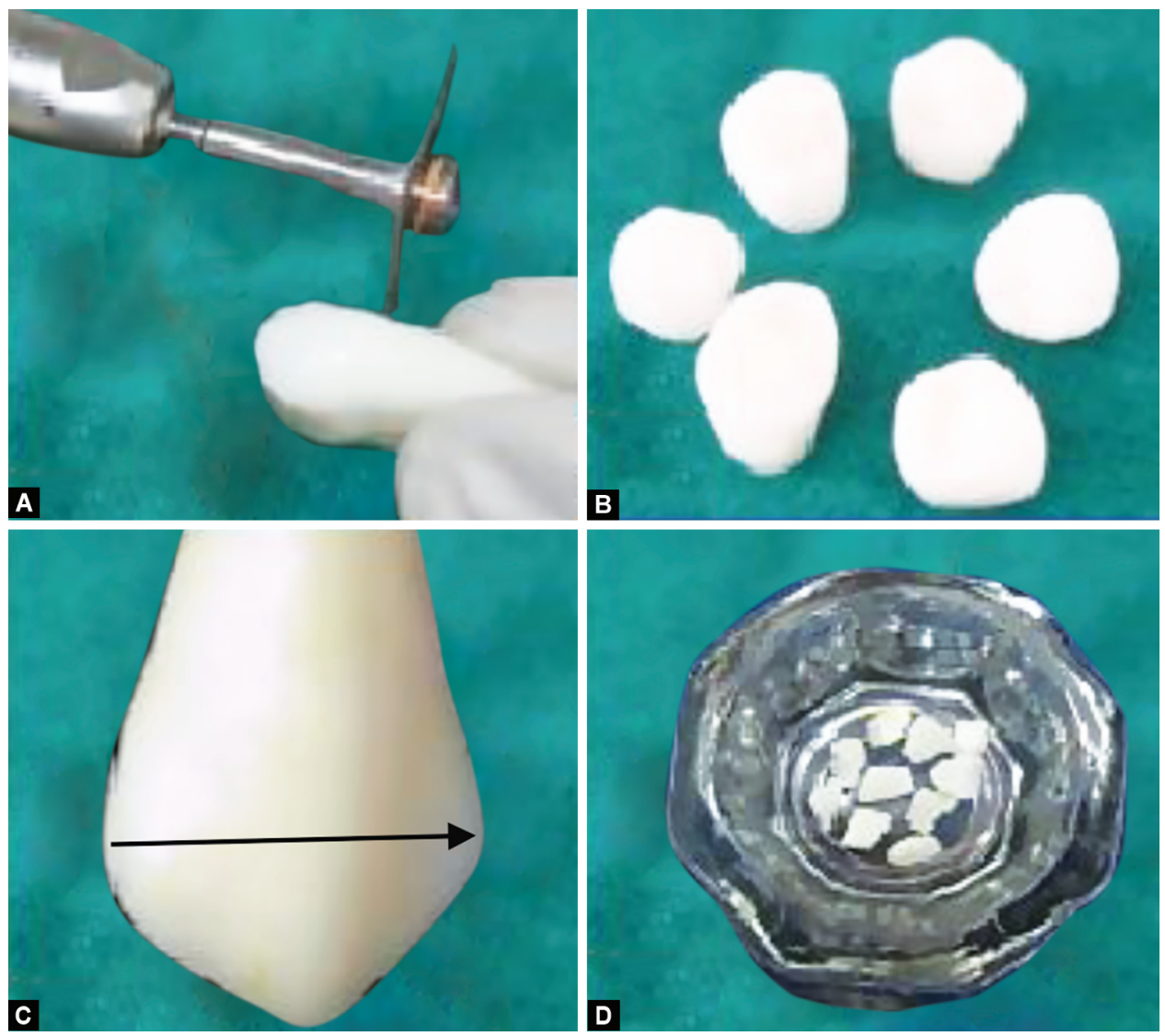

Figs 2A to D: Enamel inserts/biofillers preparation. (A) Decoronation; (B) Decoronated crowns; (C) Occlusal 3rd of crown used to prepare biofillers; (D) Enamel inserts/biofillers

Table 1: Distribution of microleakage scores in 4 groups

\begin{tabular}{|c|c|c|c|c|c|}
\hline Microleakage & Group I & Group II & Group III & Group IV & Total \\
\hline Score 0 & 0 & 2 & 3 & 4 & 9 \\
\hline Score 1 & 2 & 5 & 1 & 4 & 12 \\
\hline Score 2 & 2 & 1 & 2 & 1 & 6 \\
\hline Score 3 & 6 & 2 & 4 & 1 & 13 \\
\hline Total & 10 & 10 & 10 & 10 & 40 \\
\hline
\end{tabular}

Score: Indicates the dye penetration depth. Score 0: no penetration, Score 1: penetration $0.5 \mathrm{~mm}$, Score 2: penetration 1.0 mm, Score 3: penetration 1.5 m, Score 4: penetration pulpal wall

All the 40 samples after restoration were evaluated for microleakage under stereomicroscope.

\section{Preparation for Evaluation of Marginal Adaption Under Stereomicroscope}

The root apices of all 40 restored samples were sealed with acrylic resin. The coronal aspect of the samples was coated with two layers of nail varnish, except for the restorations and $1 \mathrm{~mm}$ of border surrounding the restorations. The specimens were thermocycled for 24 hours (approximately 860 cycles) in water baths held at $5^{\circ} \mathrm{C}$ and $55^{\circ} \mathrm{C}$. The specimens were held for 30 seconds in each bath with a transport time of approximately 20 seconds. The whole apparatus was kept at an ambient temperature of $37 \pm$ $2^{\circ} \mathrm{C}$ for the duration of thermocycling. After thermocycling, the samples were immersed in 1\% methylene blue for 4 hours, they were rinsed for 15 minutes in distilled water. The samples were dried with the help of a blotting paper to remove excess of water carefully. The roots of the teeth were cut from the crown, and a section was made through the center of the restorations at right angles to the mesiodistal plane using a slow-speed diamond saw. This created two surfaces along which dye penetration could be measured. The specimens were evaluated under 10x magnification of stereomicroscope for dye penetration along the gingival margins of class $\mathrm{V}$ restorations.

Single-blind scoring was followed. The following criteria were used to score the extent of leakage around each specimen: Score 0 : No dye penetration. Score 1: Penetration up to $0.5 \mathrm{~mm}$. Score 2: Penetration up to $1.0 \mathrm{~mm}$. Score 3: Penetration up to $1.5 \mathrm{~mm}$. Score 4: Penetration up to the pulpal wall.

Statistical evaluation of the microleakage score data was performed by Kruskal-Wallis one way analysis of variance (ANOVA) and Mann-Whitney $U$ tests. 


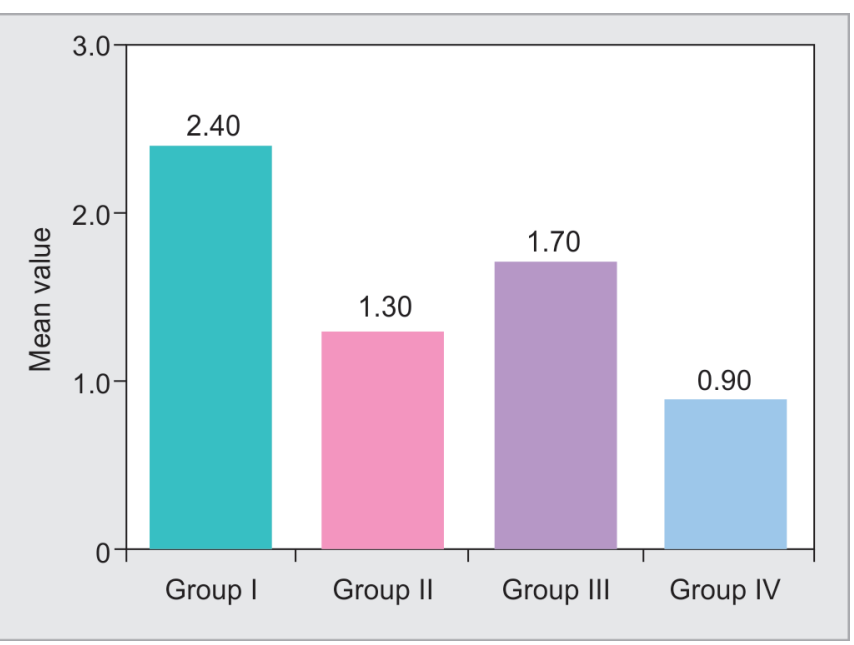

Fig. 3: Comparison of four groups with respect to mean microleakage scores

Table 2: Comparison of four groups with respect to microleakage scores by Kruskal-Wallis ANOVA

\begin{tabular}{lllll}
\hline Groups & Mean & SD & Median & $\begin{array}{l}\text { Sum of } \\
\text { ranks }\end{array}$ \\
\hline Group I & 2.4 & 0.8 & 3.0 & 284.0 \\
Group II & 1.3 & 1.1 & 1.0 & 180.0 \\
Group III & 1.7 & 1.3 & 2.0 & 215.5 \\
Group IV & 0.9 & 1.0 & 1.0 & 140.5 \\
$H$ value & 8.8142 & & & \\
$p$ value & $0.0319^{*}$ & & & \\
\hline$p<0.05$
\end{tabular}

${ }^{*} p<0.05$. Hence statistically significant

\section{Results}

Group I showed maximum dye penetration, as indicated by the scores, followed by group III and group II, respectively, least dye penetration was recorded in test group IV (Table 1). The Mean Microleakage Scores for group I (2.40) was highest followed by group III (1.70), group II (1.30), and group IV (0.90), respectively, has been plotted on Figure 3. While the distribution of microleakage scores in the four study groups is plotted on Figure 4. Kruskal-Wallis ANOVA test showed a statistically significant difference $(H$ value $=$ $8.814, p$ value $=0.03)$, between the mean microleakage scores of four groups (I to IV), presented in Table 2. Post hoc multiple pairwise comparisons of dye penetration/microleakage of the four study groups are listed in Table 3, according to Mann-Whitney $U$ test. The intergroup variations are as tabulated in Table 3. Among all pairwise comparisons of groups; groups I and II ( $p=0.0343)$, groups I and IV $(p=0.0065)$ revealed a statistically significant values.

\section{Discussion}

Failure of class $\mathrm{V}$ restorations is attributed to poor marginal adaptation. Class $V$ cavities show higher microleakage, as explained by micro-anatomic changes in the orientation and organization of the enamel rods in the cervical area, which do not favor adhesion. ${ }^{5}$ Results of this study establish that the use of biofillers in composite resins show reduced microleakage in class $V$ cavities, which is statistically significant. Highest microleakage among the four methods was seen in group I: Attributed to polymerization shrinkage. The incremental technique reduces $\mathrm{C}$-factor, reducing

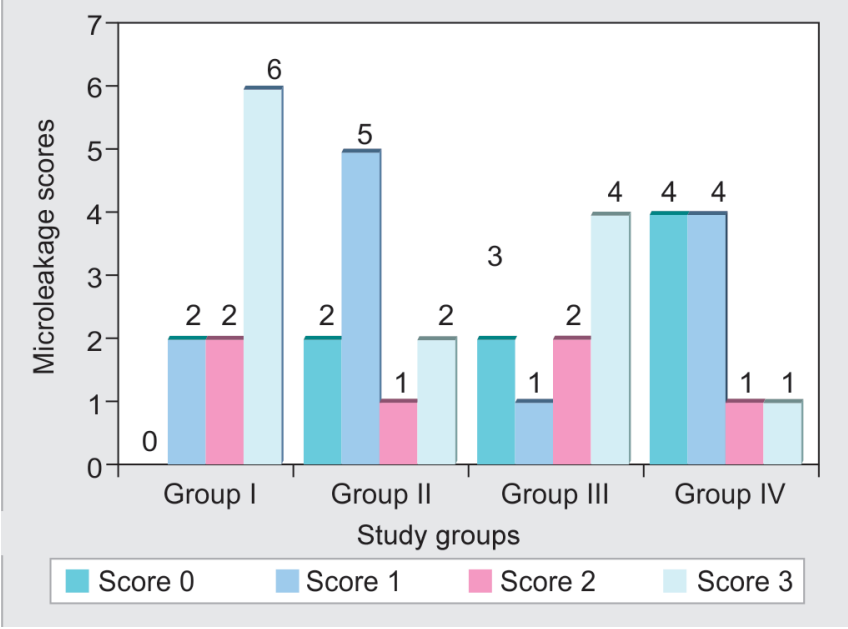

Fig. 4: Distribution of microleakage scores in the four study groups

polymerization shrinkage stress, hence group II showed less microleakage in comparison with group I and group III. While group III revealed lower microleakage compared with group I. Least microleakage was seen in group IV when compared with group III, group II, and group I.

Resin composites undergo a volumetric shrinkage of about 2-7\% during polymerization. ${ }^{6,7}$ Polymerization shrinkage affects the longevity of composite restorations more so in the class $\mathrm{V}$ cavities. The contraction stress developed due to polymerization shrinkage can cause debonding at composite/tooth interface or filler/resin interface causing failure of the restoration. The interfacial integrity of composite resins is closely related to polymerization shrinkage stress which in turn is directly related to composite volume and C-factor. ${ }^{8,9}$ Minimizing polymerization shrinkage holds the key to success of composite restorations. Decreased polymerization shrinkage translates to reduction in microgaps and less microleakage and better marginal adaptation of the composite restoration. Methods to reduce polymerization shrinkage have emphasized on either modifications to the composite material formulations or clinical procedures to reduce polymerization. ${ }^{10}$

\section{Modifications to the Composite Material ${ }^{10}$}

(1) Modification of resin matrix. (2) Modification of composite filler. (3) Modification of the photo initiator. (4) Modification of silane coupling agent.

\section{Clinical Procedures to Reduce Shrinkage Stresses ${ }^{10}$}

(1) Light-curing procedures. (2) Effect of adhesive system (adhesion). (3) Insertion technique. (4) Use of intermediate flowable layer. (5) Sealing of the outer surface. (6) Modification of dynamics of polymerization. (7) Preheating of resin composite. (8) Use of fiber insert.

In our study, of the clinical procedures to reduce shrinkage stresses, insertion techniques: Bulk method represented by group I and horizontal incremental technique in group II were utilized. Among the modifications to the composite material, modification of composite filler by incorporation of megafillers in group III and biofillers in group IV were studied. Composite placement techniques have been advocated as clinical procedures to achieve better marginal adaptation by reducing polymerization shrinkage. Results of our study revealed that horizontal incremental technique used in group II showed lower microleakage than bulk technique 
Biofillers in Class V Nanocomposite Restoration

Table 3: Pairwise comparison of four groups with respect to microleakage scores by Mann-Whitney $U$ test

\begin{tabular}{|c|c|c|c|c|c|c|c|}
\hline Groups & Mean & $S D$ & Median & Sum of ranks & Uvalue & Zvalue & pvalue \\
\hline Group I & 2.40 & 0.84 & 3.00 & 133.00 & & & \\
\hline Group II & 1.30 & 1.06 & 1.00 & 77.00 & 22.00 & -2.1166 & $0.0343^{*}$ \\
\hline Group I & 2.40 & 0.84 & 3.00 & 120.00 & & & \\
\hline Group III & 1.70 & 1.34 & 2.00 & 90.00 & 35.00 & -1.1339 & 0.2568 \\
\hline Group I & 2.40 & 0.84 & 3.00 & 141.00 & & & \\
\hline Group IV & 0.90 & 0.99 & 1.00 & 69.00 & 14.00 & -2.7213 & $0.0065^{*}$ \\
\hline Group II & 1.30 & 1.06 & 1.00 & 96.50 & & & \\
\hline Group III & 1.70 & 1.34 & 2.00 & 113.50 & 41.50 & -0.6425 & 0.5205 \\
\hline Group II & 1.30 & 1.06 & 1.00 & 116.50 & & & \\
\hline Group IV & 0.90 & 0.99 & 1.00 & 93.50 & 38.50 & -0.8693 & 0.3847 \\
\hline Group III & 1.70 & 1.34 & 2.00 & 122.00 & & & \\
\hline Group IV & 0.90 & 0.99 & 1.00 & 88.00 & 33.00 & -1.2851 & 0.1988 \\
\hline
\end{tabular}

${ }^{*} p<0.05$. Hence statistically significant

used in group I. Incremental technique reduces polymerization shrinkage by reducing C-factor, which is well established by the studies of Duarte and Saad ${ }^{11}$ and Roopa et al. ${ }^{12}$ However, few studies show conflicting reports regarding the efficacy of incremental technique as revealed by $\mathrm{Crim}^{13}$ and Santini et al. ${ }^{14}$

Variation in the material formulation, chiefly the filler loading has proved beneficial in reducing polymerization shrinkage. Nanofilled composite filler particle size ranges from 0.005 to 0.01 $\mu \mathrm{m},{ }^{15}$ reduced filler particle size allows for greater volume of filler loading without adversely affecting the mechanical properties of composite. Group III and group IV in our study represent variation in material formulation by incorporating inserts. Megafillers/ glass inserts have proven to reduce polymerization shrinkage adequately. ${ }^{16}$ Placement of one or more large fillers into the composite, is referred to as "inserts" (0.5-2 mm) or "megafiller". Inserts have been known reduce the bulk of matrix phase volume by $50-75 \%$, with a concomitant reduction in polymerization shrinkage and marginal microleakage. The concept of megafillers or inserts was extensively researched by Donly et al. ${ }^{17}$ Both ceramic and composite megafillers have been researched previously to know their role in reducing polymerization shrinkage. ${ }^{16-22}$ Precured composite balls as a megafillers in group III, increase bonding between the materials, decrease polymerization stress development due to similar physiochemical properties, and decrease the polymerization shrinkage. ${ }^{9,16}$ Thus, group III showed lower microleakage than group I. Various inserts investigated previously show the following characteristics:

Glass ceramic inserts as suggested by Salim et al. ${ }^{18}$ do not have chemical bonding to resin composites. Glass fiber inserts were the earliest inserts studied by Bowen et al., ${ }^{3}$ Donly et al. ${ }^{17}$ extensively, do not have chemical bonding to resin composites. Pre-polymerized resin inserts investigated by Wahab and Shaini ${ }^{20}$ show decreased polymerization shrinkage, but handling is difficult. Polyethylene fiber as reported by Meiers et al. ${ }^{23}$ was chemically inert, showed poor wetting and difficult bonding to composite resins. All of these inserts have inherent shortcomings because of their compositions and hence are not widely used clinically.

The presence of filler particles with hardness value greater than that of human enamel has shown to abrade the opposing enamel over time. Knoop hardness number (KHN) for: Enamel-343, Quartz-820, Silicon Carbide-2480. ${ }^{24}$ Glass-ceramic inserts may abrade the opposing enamel if they are exposed from the resin matrix. Glass-ceramic inserts also require specialized bonding methods like silanization making them expensive and timeconsuming clinically. Pre-polymerized balls show a bond strength to old and new composite: 24 to $26 \mathrm{MPa} .{ }^{25,26}$ The technique consisting of custom-made precured megafiller resin restoration, though a viable alternative for better marginal adaptation remains technique sensitive and lacks mechanical strength of human enamel.

Novel enamel inserts in group IV have proven to be the best in reducing microleakage as suggested by the results which are significant statistically. None of the previous studies have evaluated the usage of human enamel inserts in composite resins.

Enamel, a homologous tissue with better bond with resin composites, may to be better insert in terms of bonding and mechanical properties. Incorporation of filler particles with hardness characteristics similar to that of enamel may result in decreased wear and provide added advantages as listed below. (Bond strength of Enamel to composite is 28-30 MPa.) Potential Advantages of Incorporation of Enamel Inserts (Biofiller) in restoration of tooth; restorations with biofillers provide natural surface smoothness, favorable esthetics, functional masticatory efficacy. The biofillers are easily available, economical, and may also provide physiological wear resistance.

\section{Potential Disadvantages of Using Biofillers}

(1) Custom preparation of biofillers is time-consuming. (2) The use of human enamel as biofillers in restorations may not be acceptable to some patients. Limits and contraindications for the use of biofillers: (1) Patient consent is needed. (2) Extracted teeth with inadequate form, color, and structure cannot be used. (3) Mechanical properties of restorations with biofillers need to be tested.

\section{Limitations of the Study}

Custom preparation of inserts is time-consuming. Clinical trials are required to further investigate the results of our study. Dye penetration, a semi-quantitative method, was used to assess microleakage as some studies report that there was no difference between the methods regarding the evaluation of microleakage. ${ }^{19}$ However, other newer methods like radioisotope, scanning electron microscopy, and confocal microscopy may be more accurate in evaluating microleakage. ${ }^{27}$ 


\section{Conclusion}

Enamel inserts (biofillers) provide a novel approach in improving marginal adaptation of composite restorations in class $\mathrm{V}$ cavities. Incorporation of macro- and megafillers, which are capable of adequate bonding to resin and tooth, may provide improved marginal adaptability and reduce microleakage around restorative margins. Horizontal incremental technique is also an effective method in reducing microleakage and improving marginal adaptability.

\section{References}

1. Suna J, Eidelmanb N, Lin-Gibsona S. 3D Mapping of polymerization shrinkage using X-ray microcomputed tomography to predict microleakage. Dent Mater 2009(3):314-320. DOI: 10.1016/j. dental.2008.07.010.

2. Ferracane.Current JL. Trends in dental composites. Crit Rev Oral Biol Med 1995;6(4):302-318. DOI: 10.1177/10454411950060040301.

3. Bowen RL, Eichmiller FC, Marjenhoff WA. Glass-ceramic inserts anticipated for "megafiller" composite restorations. Research moves into the office. J Am Dent Assoc 1991;122(3):71-75. DOI: 10.14219/jada. archive.1991.0122.

4. Usha HL, Kumari A, Mehta D, et al. Comparing microleakage and layering methods of silorane-based resin composite in class $\mathrm{V}$ cavities using confocal microscopy: an in vitro study. J Conserv Dent 2011;14(2):164-168. DOI: 10.4103/0972-0707.82624.

5. Ferrari M, Garcia-Godoy F. Sealing ability of new generation adhesiverestorative materials placed on vital teeth. Am J Dent 2002;15:117-128.

6. Hervás-García A, Martínez-Lozano MA, Cabanes-Vila J, et al. Composite resins. A review of the materials and clinical indications. Med Oral Patol Oral Cir Bucal 2006;11:215-220.

7. Karthick K, Kailasam S, Priya PR, et al. Polymerization shrinkage of composites. A review. JIADS 2011;2:31-36.

8. Bhushan S, Logani A, Shah N. Effect of prepolymerized composite megafillers on marginal adaptation of composite restorations in cavities with different C-factors: an SEM study. Indian J Dent Res 2010;21(4):500-505. DOI: 10.4103/0970-9290.74218.

9. Takahashi H, Finger WJ, Wegner K, et al. Factors influencing marginal cavity adaptation of nanofiller containing resin composite restorations. Dent Mater 2010;26(12):1166-1175. DOI: 10.1016/j. dental.2010.08.189.

10. Magdy. AW, Gingival Microleakage of Class V Resin Composite Restorations with Fiber Inserts. (master's thesis, University of Toronto Libraries) University of Toronto; 2012.

11. Duarte SJr, Saad JR. Marginal adaptation of class 2 adhesive restorations. Quintessence Int 2008;39(5):413-419.
12. N Roopa B, Anupriya, Usha G, Karthik J, et al. Effect of four different placement technique on marginal leakage in class II composite restorations: an in vitro study. World J Dent 2011;2(2):111-116.

13. Crim GA. Microleakage of three resin placement techniques. Am J Dent 1991;4:69-72.

14. Santini A, Plasschaert A, Mitchell S. Effect of composite resin placement techniques on the microleakage of two self-etching dentin bonding agents. Am J Dent 2001;14:132-136.

15. El-Nawawy M, Koraitim L, Abouelatta O, et al. Marginal adaptation of nanofilled, packable and hybrid dental composite resins stored in artificial saliva. American Journal of Biomedical Engineering 2012;2(3):105-114. DOI: 10.5923/j.ajbe.20120203.03.

16. Mensudar R, Karthick A, Amudha D, et al. Use of innovative megafillers for improving the marginal adaptation of composite restoration. Indian J Multidisciplin Dent 2011;1(4):186-189.

17. Donly KJ, Wild TW, Bowen RL, et al. An in-vitro investigation of the effects of glass inserts on the effective composite resin polymerization shrinkage. J Dent Res 1989;68(8):1234-1237. DOI: 10.1177/00220345890680080401.

18. Salim S, Santini A, Safar KN. Microleakage around glass-ceramic insert restorations luted with a high-viscous or flowable composite. J Esthet Restor Dent 2005;17(1):30-39. DOI: 10.1111/j.1708-8240.2005. tb00080.x.

19. Sharafeddin F, Yousefi H, Modiri SH, et al. Microleakage of posterior composite restorations with fiber inserts using two adhesives after aging. J Dent Shiraz Univ Med Sci 2013;14(3):90-95.

20. Wahab FK, Shaini FJ. Evaluation of the microleakage at the proximal walls of class II cavities restored using resin composite and precured composite insert. Quint Int 2003;34:600-606.

21. Tjan AHL, Dingman TA, Woolsey BL. Microleakage of posterior composite-resin restorations using beta quartz glass-ceramic inserts. Asian J Aesthetic Dent 1993;1:81-84.

22. Strobel WO, Petschelt $A$, Kemmoona $M$, et al. Ceramic inserts do not generally improve resin composite margins. J Oral Rehabil 2005;32(8):606-613. DOI: 10.1111/j.1365-2842.2005.01459.x.

23. Meiers JC, Kazemi RB, Donadio M. The influence of fiber reinforcement of composites on shear bond strengths to enamel. J Prosthe Dentist 2003;89(4):388-393. DOI: 10.1067/mpr.2003.87.

24. O'Brien WJ. Dental material and their selection. 4th ed., Quintessence Publishing; 2008.

25. Tammy B, Davis G, Donarummo Jr J, et al. Evaluation of varied protocols applied to aged composite resin. J Adhes Dent 2005;7(1):41-49.

26. Hisamatsu N, Atsuta M, Matsumura H. Effect of silane primers and unfilled resin bonding agents on repair bond strength of prosthodontic microfilled composite. J Oral Rehab 2002;29(7):642648. DOI: 10.1046/j.1365-2842.2002.00899.x.

27. AlHabdan AA. Review of microleakage evaluation tools. J Int Oral Health 2017;9(4):141-145. 